\title{
Kairomone involvement in the host specificity of the egg parasitoid Trissolcus basalis (Hymenoptera: Scelionidae)
}

\author{
Gianandrea SALERNO ${ }^{1}$, Eric CONTI ${ }^{1}$, Ezio PERI ${ }^{2}$, Stefano COLAZZA² and Ferdinando BIN ${ }^{1}$ \\ ${ }^{1}$ D.S.A.A. - Department of Agricultural and Environmental Sciences - Entomology, University of Perugia, Borgo XX Giugno, \\ 06121 Perugia, Italy; e-mail: salerno@unipg.it \\ ${ }^{2}$ Dep. S.En.Fi.Mi.Zo., University of Palermo, Viale delle Scienze 13, 90128 Palermo, Italy
}

Key words. Scelionidae, Trissolcus basalis, egg parasitoid, Pentatomidae, kairomone, host selection, host specificity

\begin{abstract}
This paper reports the results of a comparative laboratory analysis of the behavioural responses of the egg parasitoid Trissolcus basalis (Wollaston) (Hymenoptera: Scelionidae) to semiochemical cues from four species of pentatomid bugs, Nezara viridula (L.), Eurydema ventrale Klt., Murgantia histrionica Hahn. and Graphosoma semipunctatum F. (Heteroptera: Pentatomidae). In a Y-tube olfactometer, T. basalis was attracted by volatile chemicals from N. viridula, but not from other pentatomid species. In an open arena, the parasitoid reacted to chemical trails left on filter paper by all the species but most intensely to those left by $N$. viridula. However, upon encountering pentatomid eggs, T. basalis examined more intensely and probed more frequently the eggs of $G$. semipunctatum than those of the other species. The parasitoid only parasitized and emerged from eggs of G. semipunctatum and $N$. viridula; those of the other species were unsuitable. Therefore $N$. viridula is semiochemically confirmed to be a coevolved host (old association) of T. basalis, whereas G. semipunctatum may be a potential non-coevolved host (new association). The utility of these tests for defining a parasitoids' host specificity and in assessing the risk of non-target effects in biological control is discussed.
\end{abstract}

\section{INTRODUCTION}

Insect parasitoids are important indicators of species richness and are also often used as models for studies on behavioural and chemical ecology. Female parasitoids forage for host insects to provide food for their offspring. Female parasitoid foraging behaviour has been divided into a series of hierarchical steps: habitat preference, host community location, host location, host recognition and acceptance, and among the different ecological and physiological factors involved, chemical cues influence all of these steps (Vet \& Dicke, 1992; Vinson, 1998; Steidle \& van Loon, 2002). When dealing with host - egg parasitoid associations, stimuli indirectly associated with the presence of the host (Nordlund, 1994; Schmidt, 1994; Vinson, 1998; Powell, 1999) and coming from the plant host complex (Hilker \& Mainers, 2002; Hilker et al., 2002) play a fundamental role.

Recent research has shown the importance of host semiochemicals in determining host specificity in parasitoids (Meiners et al., 2000; Conti et al., 2004). Such studies suggest new ways of evaluating and predicting the possible adverse side effects of biological control agents on non-target species (Conti et al., 2004). Quantifying the specificity of host-parasitoid associations is necessary for safe and successful biological control (Hågvar, 1991; Nechols et al., 1992; FAO, 1995; van Lenteren, 1997; Legner \& Bellows, 1999; van Lenteren et al., 2003). Basic information on the level of specificity of a natural enemy can be obtained from host records in the literature (Nechols et al., 1992; Gordh \& Beardsley, 1999) and sequential specificity tests (van Lenteren et al., 2003). However, host records are often poor and/or unreliable (Nechols et al., 1992; Gordh \& Beardsley, 1999), whereas host specificity tests, although a fundamental tool for the practitioner, do not explain the mechanisms that determine host range. In contrast, studies of the chemical cues involved in host/prey selection behaviour (Vet \& Dicke, 1992; Vinson, 1998) provide a quantifiable characterization of host specificity.

Trissolcus basalis (Wollaston) (Hymenoptera: Scelionidae), an egg parasitoid of the southern green stink bug, Nezara viridula (L.) (Heteroptera: Pentatomidae), responds to volatile chemicals and the chemical trails left by adult hosts (i.e., chemicals remaining on the substrate where the host has walked) during host searching (Colazza et al., 1999), even though adults are not the stage it parasitizes. In addition, T. basalis responds to volatile plant cues systemically induced by $N$. viridula oviposition and feeding (Colazza et al., 2004a, b). The host-induced plant volatiles and adult volatiles are probably used to locate a potential host community, whereas the chemical trails left by adults and nymphs elicit parasitoid arrestment and stimulate searching. Finally, the process continues with host recognition, which is mediated by contact chemicals present in the ovariole secretion used by the host as an egg adhesive (Bin et al., 1993; Conti et al., 2003). Comparable cues are used in other Trissolcus spp. - Pentatomid associations such as Trissolcus simoni (Mayr) - Eurydema ventrale Kolenati (the European harlequin bug) (Conti et al., 2003; Colazza \& Bin, pers. observ.) and Trissolcus brochymenae (Ashmead) - Murgantia histrionica Hahn (the American harlequin bug), where the parasitoid also responds to volatile and chemical trails from nymphs and short-range volatiles from the host's eggs (Conti et al., 2003). 
According to the literature, T. basalis is associated with at least 51 species of pentatomid bugs (Salerno, 2000). In spite of its broad host range, this parasitoid has been used on a world-wide scale, with different degrees of success, for biological control of $N$. viridula (Jones, 1988; Jones et al., 1996), a serious pest of more than 30 different crops (Todd, 1989).

However, although many crops are also attacked by other pentatomid bugs, T. basalis may not parasitize all species. For instance, on cabbage and other Cruciferae this parasitoid parasitizes the southern green stink bug but not the European harlequin bug (N.F. Johnson \& L. Musetti, Hymenoptera On-Line Database, http://iris.biosci. ohio-state.edu/hymenoptera/; Salerno, 2000). In the USA, however, T. basalis is occasionally recorded from the American harlequin bug (Buschman \& Whitcomb, 1980). Conversely, although T. basalis has never been recorded from Graphosoma semipunctatum (F.) (Heteroptera: Pentatomidae) (N.F. Johnson \& L. Musetti, Hymenoptera On-Line Database, http://iris.biosci. ohio-state.edu/hymenoptera/; Salerno, 2000) in the field, this parasitoid can be successfully mass reared on $G$. semipunctatum eggs (Kartavtsev et al., 1975). Therefore, the question to be addressed here is: could such aspects of host specificity be explained in terms of semiochemical cues (host kairomones) influencing the different steps in host selection?

A series of experiments were designed to verify the host records reported in literature and understand how an incomplete host selection sequence may interfere with a given host-parasitoid association. The behavioural responses of $T$. basalis to chemical cues from four pentatomids: (1) the co-evolved host, N. viridula, (2) a nonhost such as E. ventrale, (3) an occasional allopatric host such as $M$. histrionica and (4) a laboratory host such as $G$. semipunctatum, were compared. In particular, $T$. basalis' sequential responses to chemical volatiles and trails produced by gravid females (host location), and to chemical and physical factors associated with the host eggs (host recognition) and with the accepted host (host suitability) were determined.

\section{METHODS}

\section{Insect cultures}

The cultures of $N$. viridula, E. ventrale and G. semipunctatum were obtained from individuals collected on wild and cultivated host plants in the area of Perugia, central Italy. M. histrionica was collected from cabbage in the Beltsville area, Maryland, USA (kindly provided by Jeff Aldrich). Both nymphs and adults of the four species were reared separately, under controlled conditions $\left(25 \pm 2{ }^{\circ} \mathrm{C}, 70 \pm 10 \% \mathrm{RH}, 14 \mathrm{~L}: 10 \mathrm{D}\right.$ photoperiod), inside clear plastic food containers $(300 \times 195 \times 125 \mathrm{~mm}$-high $)$ with 5 -cm diameter mesh-covered holes. All stages were fed with seeds, fruits and vegetative parts of their preferred food, which was changed every $2-3$ d. French beans (Phaseolus vulgaris L.) and sunflower seeds (Helianthus annuus L.) were used to feed N. viridula, fennel (Phoeniculum vulgare Miller), Ferula communis L., and seeds of celery (Apium graveolens L.) and parsley (Apium petroselium L.) to feed G. semipunctatum, cabbage and broccoli (Brassica oleracea L.) to feed E. ventrale and M. his- trionica. Water was provided weekly in soaked cotton wool balls.

T. basalis was collected from fields in the area of Perugia, Italy, using $N$. viridula sentinel egg clusters; i.e., laboratory laid egg clusters were exposed on plants of French bean, maize and fava bean in the field. The parasitoid was reared in $85-\mathrm{ml}$ glass tubes ( $30 \mathrm{~mm}$ diameter $\times 150 \mathrm{~mm}$ length) and kept in an incubator $\left(25 \pm 1^{\circ} \mathrm{C}, 80 \pm 5 \% \mathrm{RH}, 15 \mathrm{~L}: 9 \mathrm{D}\right.$ photoperiod). Adult wasps were fed on a specific parasitoid diet (Safavi, 1968). Freshly laid (0-24 h old) host eggs were exposed to parasitoid females for $48 \mathrm{~h}$. Females were then removed and the eggs were stored for incubation. After emergence, male and female parasitoids were kept together to allow mating. Twenty-four $\mathrm{h}$ before the experiments were started, $2-5 \mathrm{~d}$ old mated females were isolated in small glass vials (10 diameter $\times 25 \mathrm{~mm}$ length) containing a small drop of the Safavi diet and kept in an incubator $\left(25 \pm 1{ }^{\circ} \mathrm{C}, 80 \pm 5 \% \mathrm{RH}, 15 \mathrm{~L}: 9 \mathrm{D}\right.$ photoperiod).

\section{Parasitoid response to volatile chemical cues in a Y-tube olfactometer}

The response of $T$. basalis females to volatile chemicals from pentatomid bugs was evaluated in a Y-tube olfactometer, which consisted of a polycarbonate plate (15 mm thickness) with a Y-shaped space milled into the centre (stem $90 \mathrm{~mm}$ long; arms $80 \mathrm{~mm}$ long at $130^{\circ}$ angle; internal section $15 \times 15 \mathrm{~mm}$ ) sandwiched between two glass plates. A $10 \mathrm{~mm}$ diameter hole was drilled through the block into the end of each arm to allow air tubes to be connected and the test organisms to be introduced. Compressed air of medical-grade, regulated by flowmeters, ran through both arms creating an air stream of $144 \mathrm{ml} / \mathrm{min}$ per arm. To humidify the air before it passed into the olfactometer, the flow was bubbled through a water jar. The olfactometer was illuminated by two $22-\mathrm{W}$ cool white fluorescent tubes located above the device, which to minimize possible cues from the room was surrounded by a black curtain. The temperature in the bioassay room was maintained at $\approx 25^{\circ} \mathrm{C}$.

During host location, the parasitoid $T$. basalis uses cues from different host instars, but shows a stronger response to gravid females (Colazza et al., 1999). Therefore, such pentatomid females, which can be recognized by their enlarged and slightly bloated abdomens, were bioassayed. For each experiment, one female was caged in a small brass mesh box $(25 \times 15 \mathrm{~mm})$ and placed close to the air orifice at the end of one arm, randomly assigned. An empty box was placed at the end of the control arm. Each species (N. viridula, E. ventrale, M. histrionica or $G$. semipunctatum) was used in a set of 4-5 bioassays, each carried out with a different parasitoid female. After every set of trials the glass plates and brass mesh boxes were cleaned with hexane, acetone, ethanol and distilled water, and the polycarbonate part of the olfactometer was cleaned with a laboratory detergent, rinsed first with hot tap water $\left(\approx 90^{\circ} \mathrm{C}\right.$, for $\left.5 \mathrm{~min}\right)$ and finally with distilled water.

The possible presence of bias between the two olfactometer arms was analysed by running blank tests, i.e., tests with empty arms, and all tests were conducted between $\approx 9: 00$ am and 5:00 pm.

A naive female of $T$. basalis was used in each experiment. The parasitoid was introduced into the end of the central arm of the olfactometer and its walking pattern was then recorded for 10 min using a monochrome CCD videocamera (Sony SSC M370 CE) fitted with a $12.5-75 \mathrm{~mm} / \mathrm{F} 1.8$ zoom lenses. In order to exclude reflected light, the olfactometer was illuminated by infra-red light (homogenous emission of wavelengths at $950 \mathrm{~nm}$ provided by 108 LEDs) from below and the camera lens was covered with an infrared pass filter (Kodak Wratten filter $87 \AA$ ). Analogue video signals from the camera were digit- 
ized by a video frame grabber and the digital data processed (sample rate $=\sim 8$ images $/ \mathrm{sec}$ ) by XBug, a video tracking and motion analysis programme developed for the Linux operating system (Peri et al. unpublished data).

Among the different parameters computed by the system, those that best describe the parasitoid walking patterns were analysed. These are the percentage residence time, i.e., the percentage of total time spent in each of the olfactometer arms (\%), and the average linear speed measured in each arm $(\mathrm{mm} / \mathrm{sec})$.

\section{Parasitoid response to chemical trails in an open arena}

The parasitoid response to chemical trails left by the different bug species was tested using a filter paper open arena $(380 \times$ $252 \mathrm{~mm}$ ), the central circular area (58 $\mathrm{mm}$ of diameter) of which was contaminated by a gravid host female. Uncontaminated arenas were also used as controls.

In order to contaminate the arena, a pentatomid bug female was kept in place for $1 \mathrm{~h}$ and forced to walk on the filter paper surface using a special device made from a transparent polyethylene Petri dish cover connected to a small clock. The axis of the clock was introduced through a hole in the middle of the cover and only the second hand left connected. The movement of the second hand forced the host to move continuously and uniformly in the constrained area.

A naive female of $T$. basalis was also used for each experiment. A female parasitoid was placed in the middle of the central area, either host-contaminated or uncontaminated, and its walking behaviour recorded. The experiment was stopped when the female either walked or flew out of the arena. Four to five bioassays were conducted on each arena, then the whole device was replaced and the process repeated. The parasitoid's walking pattern was recorded using a video camera and the digitized data processed using XBug, as described for the olfactometer bioassays.

As the experiments were conducted in an open arena, the parasitoids were unconstrained. Therefore it was possible to consider behavioural parameters other than those recorded in the olfactometer bioassays. The parameters, used to characterize the parasitoid walking patterns on the host-contaminated patch, were: (1) total residence time (sec); (2) average linear speed $(\mathrm{mm} / \mathrm{sec})$; (3) average angular speed $(\% / \mathrm{sec}) ;(4)$ tortuosity index. The tortuosity index ranges from 0 to 1 , with 0 indicating a completely linear track and 1 the maximum tortuosity. This index was computed as follows (Peri et al., unpubl. data):

$$
T=1-m p / t l
$$

with $T$, tortuosity index; $m p$, maximum projection of the track over the generic line in the plan; and $t l$ the total length of the track.

\section{Parasitoid response to egg cues in a confined arena}

The egg parasitoid $T$. basalis recognizes the eggs of its host by means of physical factors and a host recognition kairomone, which is in the follicular cell secretion used by a female bug to glue her egg mass onto the substrate (Bin et al., 1993). The parasitoid response (i.e., host recognition and acceptance behaviour) to the eggs of the different bug species were compared. The experiments were conducted in multiple, confined, arenas by exposing a simplified egg cluster (i.e., three eggs) of either $N$. viridula, E. ventrale, M. histrionica or G. semipunctatum to a 2- to 5-d-old, naive, female parasitoid. The arenas were shaped from a polycarbonate plate $(80 \times 60 \times 4 \mathrm{~mm}$ thick $)$ with 6 holes, each acting as single arena ( $4 \mathrm{~mm}$ height $\times 13 \mathrm{~mm}$ diameter), sandwiched between two glass plates. The eggs were placed in the centre of each arena and a tunnel $(3 \mathrm{~mm}$ diameter, $10 \mathrm{~mm}$ length) in the external wall of each arena was used to introduce a parasitoid female. The observations were started after parasi- toid introduction and stopped after $10 \mathrm{~min}$ if no encounter occurred, $15 \mathrm{~min}$ after an encounter if there was no recognition, or after parasitization. Only observations that led to an encounter were recorded and analysed. The parasitoid behaviour was recorded with a JVC KY-M280 video camera using 12.5-75 mm / F1.8 lenses and connected to a Panasonic NVFS100HQ video recorder. Behavioural data were then collected and analyzed using The Observer Video-Pro Version 4.0 for Windows (Noldus Information Technology, 1997). The following parasitoid behaviours were analysed: encounters with host eggs (frequency); examination of host eggs with antennae (frequency and duration); staying motionless (frequency and duration); grooming of antennae and other body parts (frequency and duration); probing of host egg with the ovipositor (percentage of parasitoids probing and duration); oviposition into the host egg (percentage of parasitoids ovipositing), which was scored every time a parasitoid showed marking behaviour, which indicates that an egg has definitely been parasitized (Colazza et al., 1996).

The suitability of pentatomid eggs for parasitoid development was evaluated by calculating the emergence from eggs that were parasitized. Because this work focused on parasitoid responses to the chemosensory cues elicited by bugs, the parameters for evaluating host suitability, such as brood size, fecundity and behaviour were not measured.

\section{Data analysis}

The data on parasitoid response to volatile cues, in the treatment $v s$. control arms of the olfactometer, were analysed with Wilcoxon tests for paired comparisons. The same statistical test was used to evaluate the possible presence of bias in the blank tests by comparing the two arms of the olfactometer. To analyse the changes in parasitoid walking behaviour in response to host trails in the open arena, the Kruskall Wallis Anova and the Dunn's test for multiple comparisons were used (Siegel \& Castellan, 1988; Statistica, 1997; Zar, 1999). For the parasitoids response to host eggs in confined arenas, the Kruskall Wallis Anova and the Dunn's test for multiple comparisons were used to analyse frequencies $(\mathrm{N})$ and mean durations (s) of behavioural steps, and the Pearson Chi-square test and the Goodman's post hoc procedure were used for internal contrasts between bug species to compare frequencies of probing and ovipositing in the host eggs (\% of females showing such behaviour) (Siegel \& Castellan, 1988; Zar, 1999). Finally, the percentages of parasitoid emergence were compared using the Chi-square test. The rejection probability $(\mathrm{P})$ was set at 0.05 for each test used.

\section{RESULTS}

The egg parasitoid T. basalis responded in the olfactometer, open arena and confined arena, to cues of its coevolved host $N$. viridula, and cues of some other bug species. A synthesis of $T$. basalis responses to cues mediating the host selection of $N$. viridula, E. ventrale, M. histrionica and G. semipunctatum is shown in Fig. 4, and the details in Figs 1-3.

\section{Parasitoid response to volatile chemical cues}

Trissolcus basalis exhibited a clear preference for the volatile cues produced by gravid $N$. viridula, as the residence time was significantly longer $(\mathrm{T}=21, \mathrm{~N}=20, \mathrm{P}<$ $0.002)$ and linear speed significantly lower $(\mathrm{T}=8, \mathrm{~N}=$ $18, \mathrm{P}<0.001)$ in the olfactometer arm containing the host compared with the control arm (Figs 1A, B). The reduction in linear speed suggests an increase in searching behaviour in the area contaminated by host volatiles. 

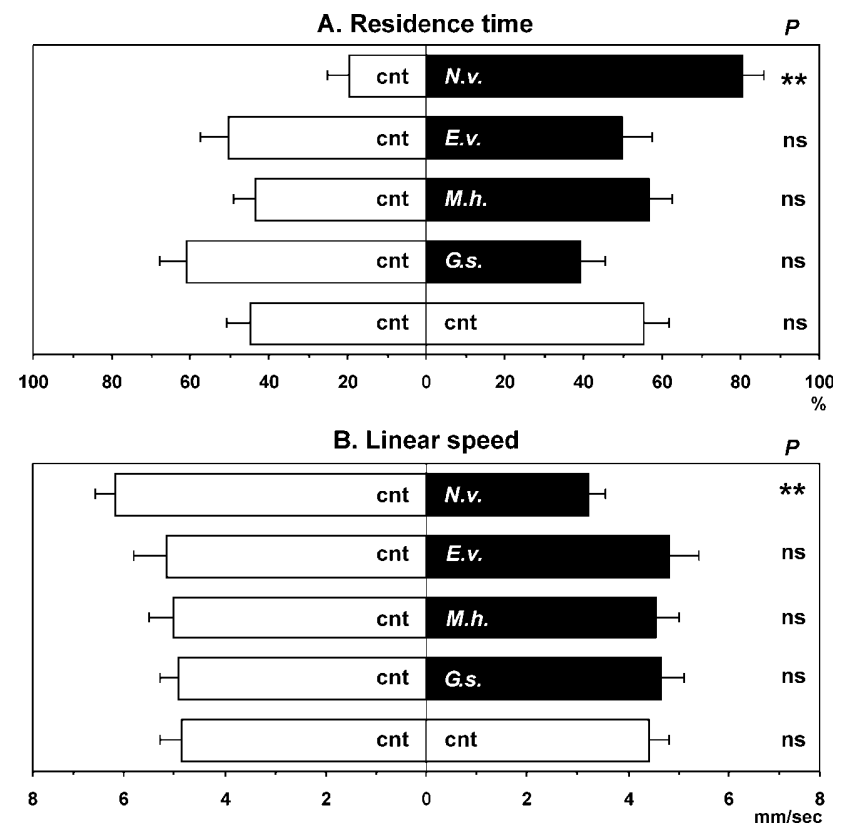

Fig. 1. Behavioural responses (A - residence time; $\mathrm{B}$ - linear speed) (mean $\pm \mathrm{SE}$ ) of the parasitoid Trissolcus basalis in a Y-tube olfactometer to volatile cues from its co-evolved host, Nezara viridula (N.v.) $(\mathrm{n}=20)$, and from Eurydema ventrale (E.v.) $(\mathrm{n}=14)$, Murgantia histrionica $($ M.h. $)(\mathrm{n}=15)$ and Graphosoma semipunctatum (G.s.) $(\mathrm{n}=17)$; control (cnt) $(\mathrm{n}=16)$. ** $\mathrm{P}<0.01$; ns - not significant (Wilcoxon test for paired comparisons).

However, T. basalis did not show any significant differences between arms when $E$. ventrale (Residence time: T $=52, \mathrm{~N}=14, \mathrm{P}=0.975$. Linear speed: $\mathrm{T}=44, \mathrm{~N}=14, \mathrm{P}$ $=0.594), M$. histrionica (Residence time: $\mathrm{T}=33, \mathrm{~N}=15$, $\mathrm{P}=0.125$. Linear speed: $\mathrm{T}=28, \mathrm{~N}=15, \mathrm{P}=0.069)$ or $G$. semipunctatum (Residence time: $\mathrm{T}=46, \mathrm{~N}=17, \mathrm{P}=$ 0.149. Linear speed: $\mathrm{T}=49, \mathrm{~N}=15, \mathrm{P}=0.532$ ) were tested, or in the blank (Residence time: $\mathrm{T}=48, \mathrm{~N}=16, \mathrm{P}$ $=0.301$. Linear speed: $\mathrm{T}=52, \mathrm{~N}=16, \mathrm{P}=0.408)$. The latter indicates that there was no bias in the olfactometer (Figs 1A, B).

\section{Parasitoid response to chemical trails}

The parasitoid females exhibited a clear preference in open arenas for substrates contaminated with trails of its co-evolved host, $N$. viridula, compared with those contaminated by other, non co-evolved, pentatomid bugs (Figs 2A-D).

Residence time was significantly higher in the $N$. viridula contaminated arenas compared to those contaminated by non-coevolved bugs, where it was intermediate, and to the control. However, the residence time was higher in the arenas contaminated by the three noncoevolved species compared to the control, with M. histrionica eliciting a stronger response than either $E$. ventrale or G. semipunctatum $[\mathrm{H}(4, \mathrm{n}=182)=68.24, \mathrm{P}<$ 0.0001] (Fig. 2A).

Linear speed was significantly lower on patches contaminated by bugs compared to uncontaminated patches, except for G. semipunctatum. In detail, the linear speed
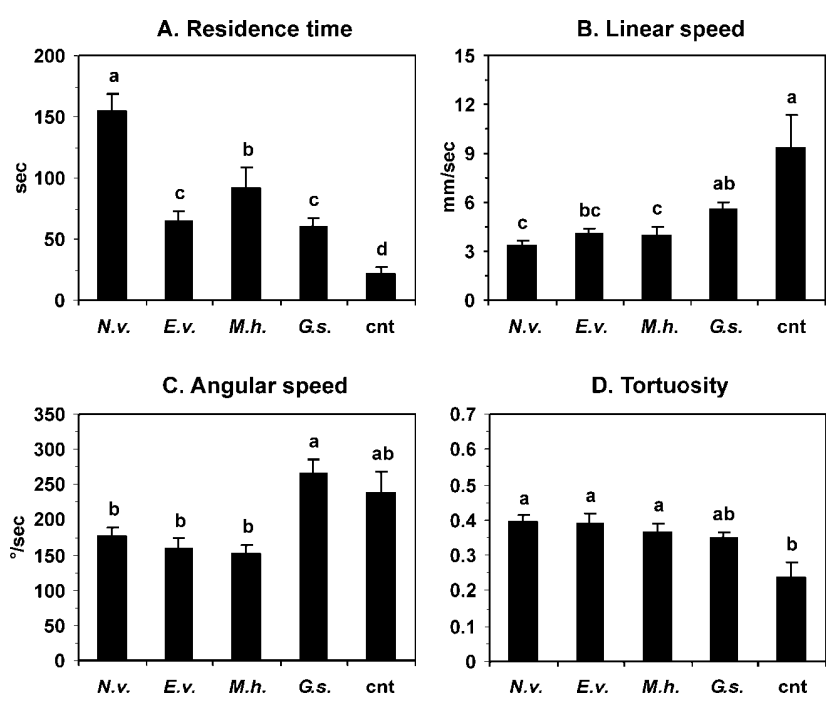

Fig. 2. Behavioural responses (A - residence time; $\mathrm{B}$ - linear speed; C - angular speed; D - tortuosity) (mean $\pm \mathrm{SE}$ ) of the parasitoid Trissolcus basalis in an open arena to chemical trails of its co-evolved host, Nezara viridula (N.v.) $(\mathrm{n}=36)$, and of Eurydema ventrale (E.v.) $(\mathrm{n}=38)$, Murgantia histrionica (M.h.) $(\mathrm{n}=46)$ and Graphosoma semipunctatum (G.s.) $(\mathrm{n}=31)$; control (cnt) $(\mathrm{n}=30)$. Columns with the same letter are not significantly different at $\mathrm{P}<0.05$ (Kruskall-Wallis Anova, Dunn's test).

was lowest in arenas contaminated by $N$. viridula, M. histrionica and $E$. ventrale, and of an intermediate value in those contaminated by $G$. semipunctatum $[\mathrm{H}(4, \mathrm{n}=182)$ $=34.70, \mathrm{P}<0.0001]$ (Fig. 2B).

There were no significant differences in the angular speed of the parasitoid on bug contaminated patches vs. the control. However, this variable was significantly higher on the $G$. semipunctatum contaminated patches than on those contaminated by the other bug species $[\mathrm{H}$ $(4, \mathrm{n}=182)=24.12, \mathrm{P}<0.001]$ (Fig. 2C).

Tortuosity was significantly higher on the patches contaminated by $N$. viridula, E. ventrale and $M$. histrionica compared with the control, and intermediate in those contaminated by $G$. semipunctatum $[\mathrm{H}(4, \mathrm{n}=182)=21.83, \mathrm{P}$ $<0.001]$ (Fig. 2D).

\section{Parasitoid response to egg cues}

Surprisingly, in the confined arenas, Trissolcus basalis responded more strongly to the eggs of $G$. semipunctatum than to those of its co-evolved host, $N$. viridula (Figs $3 \mathrm{~A}-\mathrm{G})$.

The frequencies of encounters $[\mathrm{H}(3, \mathrm{n}=127)=62.42$, $\mathrm{P}<0.0001]$ and examinations $[\mathrm{H}(3, \mathrm{n}=127)=58.47, \mathrm{P}$ $<0.0001]$ were similar for eggs of $N$. viridula, E. ventrale and $M$. histrionica but higher than those of G. semipunctatum. Instead, the duration of examination $[\mathrm{H}(3, \mathrm{n}=$ $126)=36.25, \mathrm{P}<0.0001]$ was longer for eggs of $G$. semipunctatum than those of the other species (Figs 3A, B). Staying frequency $[\mathrm{H}(3, \mathrm{n}=127)=7.26, \mathrm{P}=0.064]$ and duration $[\mathrm{H}(3, \mathrm{n}=127)=5.27, \mathrm{P}=0.153]$ did not show significant differences (Figs $3 \mathrm{C}$ ). Grooming frequency $[\mathrm{H}$ $(3, \mathrm{n}=127)=43.51, \mathrm{P}<0.0001]$ and duration $[\mathrm{H}(3, \mathrm{n}=$ $102)=15.64, \mathrm{P}=0.001]$ were lower for $G$. semipunc- 


\section{A. Encounter}

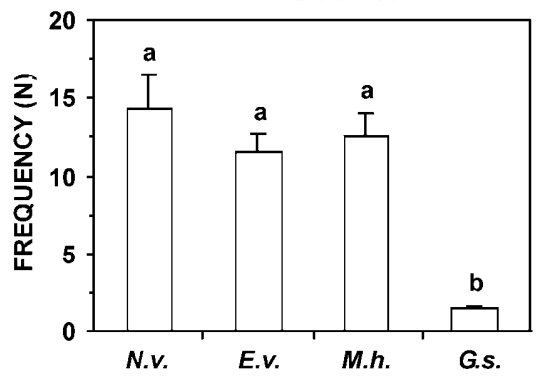

C. Staying

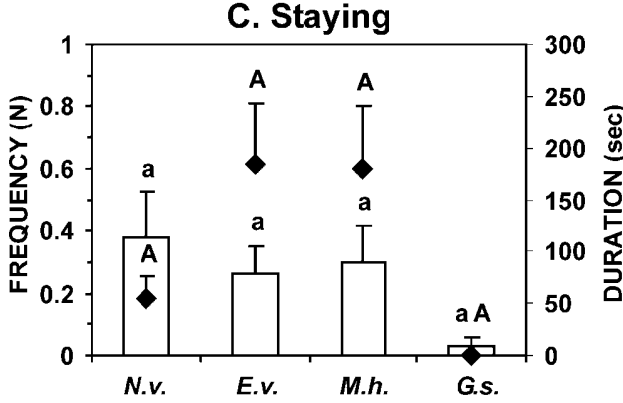

E. Probing

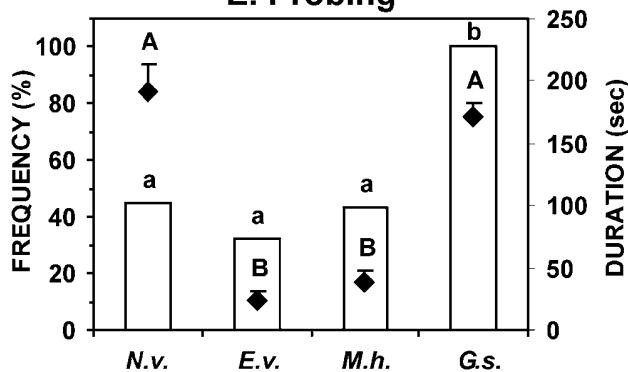

B. Examination

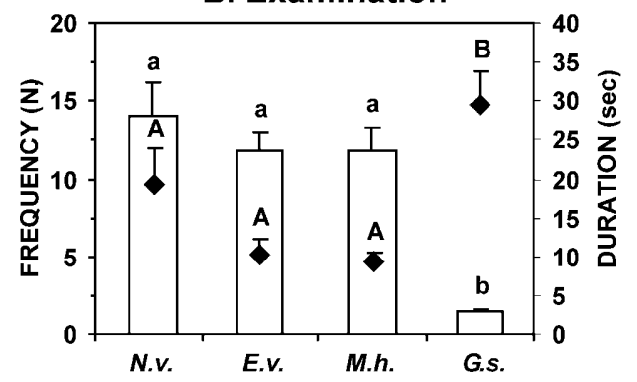

D. Grooming

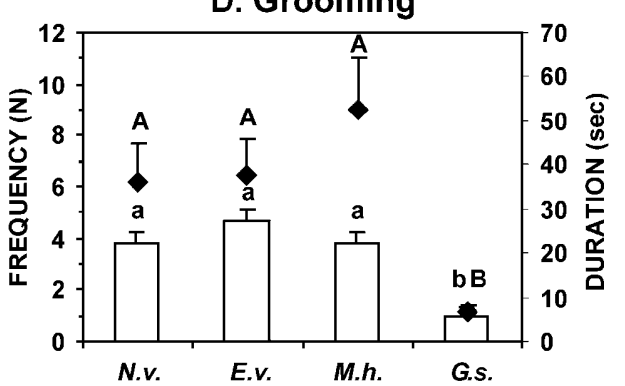

F. Oviposition

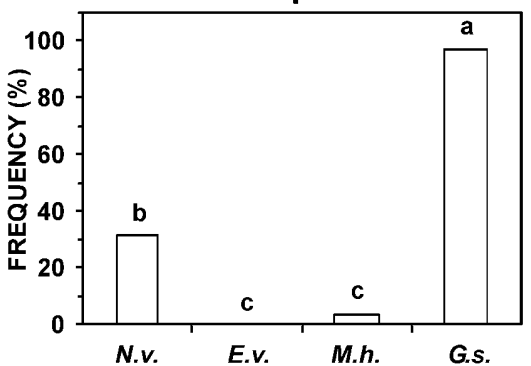

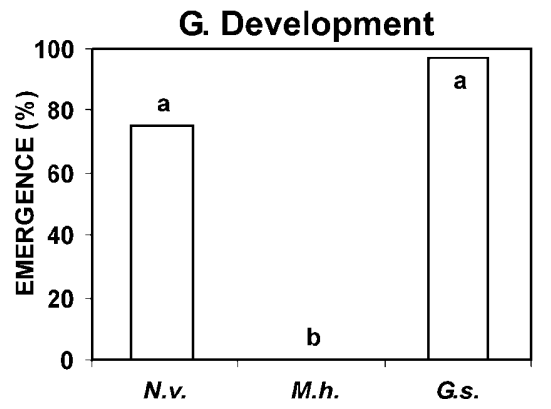

Fig. 3. Behavioural responses (A - encounter; B - examination; $\mathrm{C}$ - staying; $\mathrm{D}$ - grooming; $\mathrm{E}$ - probing; $\mathrm{F}$ - oviposition; $\mathrm{G}$ development) (mean $\pm \mathrm{SE}$ ) of the parasitoid Trissolcus basalis in a confined arena to eggs of its co-evolved host, Nezara viridula (N.v.) (n=29), and of Eurydema ventrale (E.v.) $(\mathrm{n}=34)$, Murgantia histrionica (M.h.) $(\mathrm{n}=30)$ and Graphosoma semipunctatum (G.s.) $(\mathrm{n}=34)$. The columns show behavioural frequencies, the dots the duration of the various behaviours. Columns with the same letter do not differ significantly at $\mathrm{P}<0.05$ (Kruskall-Wallis Anova, Dunn's test; Karl Pearson $\chi^{2}$ test).

tatum eggs than for those of the other three species (Figs 3D). The highest percentage of probing by T. basalis $\left(\chi^{2}\right.$ $=37.84, \mathrm{df}=3, \mathrm{P}<0.0001)$ was recorded for $G$. semipunctatum eggs, whereas the duration $[\mathrm{H}(3, \mathrm{n}=71)=$ $40.70, \mathrm{P}<0.0001]$ was higher for $N$. viridula and $G$. semipunctatum eggs compared to those of $E$. ventrale and M. histrionica (Figs 3E). The percentage oviposition $\left(\chi^{2}=\right.$ 90.63, df $=3, \mathrm{P}<0.0001)$ was highest for $G$. semipunctatum eggs, second highest for those of $N$. viridula and negligible for $M$. histrionica; E. ventrale eggs were never accepted (Fig. 3F).

Trissolcus basalis developed normally in the eggs of its co-evolved host, $N$. viridula, and in those of G. semipunctatum, but never emerged from the few $M$. histrionica eggs that were accepted $\left(\chi^{2}=36.98, \mathrm{df}=2, \mathrm{P}<0.0001\right)$ (Fig. 3G). 


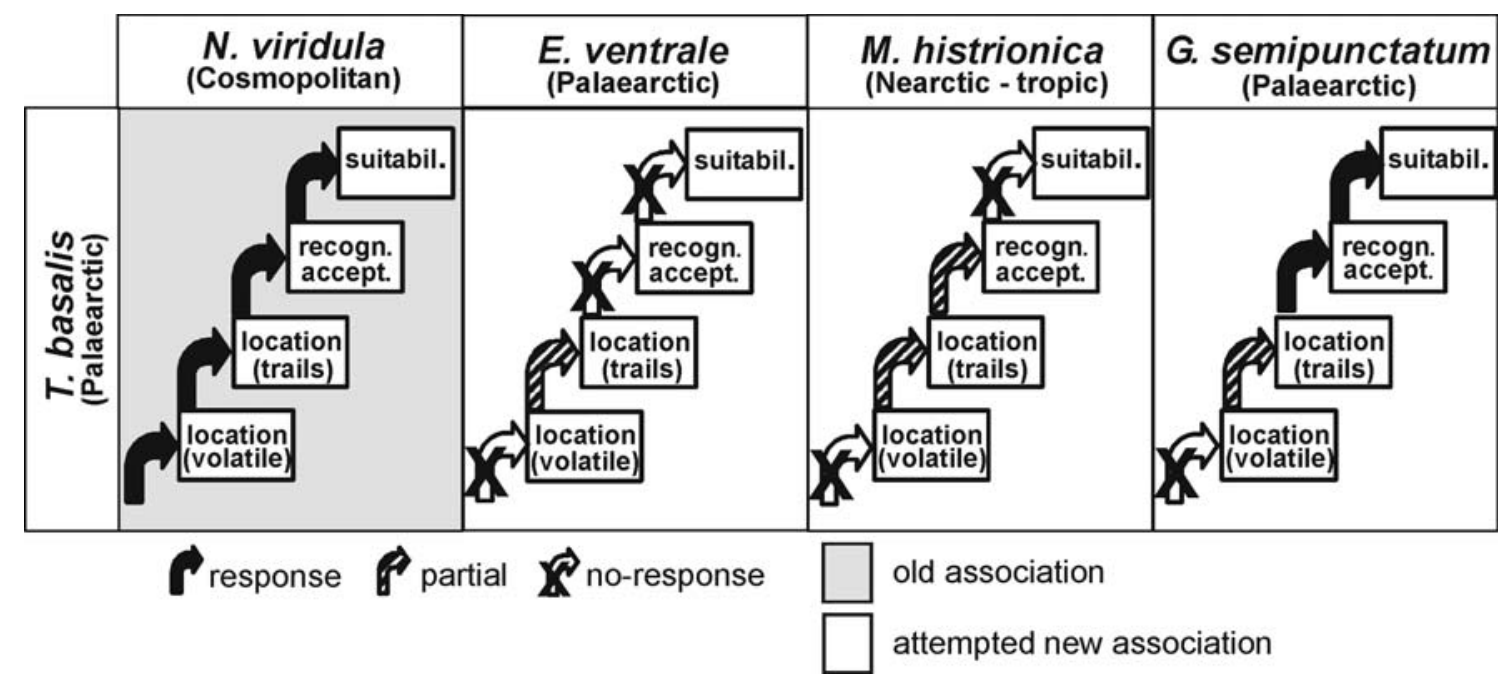

Fig. 4. Diagrammatic representation of Trissolcus basalis responses, during the different host selection steps, elicited by cues from Nezara viridula, Eurydema ventrale, Graphosoma semipunctatum and Murgantia histrionica. Location (volatile): host location elicited by volatile cues. Location (trails): host location mediated by chemical trails. Recogn. accept.: egg recognition and acceptance indicated by probing and oviposition, respectively. Suitabil.: egg suitability as indicated by parasitoid development. For details see Figs $1-3$.

\section{DISCUSSION}

The results of our experiments provide new insights into the host-parasitoid associations of T. basalis, which would be useful for comparing with the records in the literature and for checking the reliability of host records. Furthermore, our results indicate how the lack of one or more steps in the host selection process (Fig. 4) could interfere with a given association in the field, although such association may occur in the laboratory. This confirms that parasitoid specificity may be affected by response to cues involved in host selection (Vet \& Dicke, 1992; Meiners et al., 2000; Steidle \& van Loon, 2003; Conti et al., 2004).

In our experiments the cues that originate from the host (kairomones) and seem to be used by most Trissolcus spp. associated with Pentatomidae were tested. This comparative analysis confirms the association in the field of $T$. basalis with its co-evolved host $N$. viridula reported in the literature, as in the laboratory all the steps necessary for a complete parasitoid response were present (Fig. 4). However, the field association T. basalis $-M$. histrionica, reported from Florida (Buschman \& Whitcomb, 1980), was not confirmed by our laboratory tests. In fact, $T$. basalis did not develop in eggs of $M$. histrionica, neither responded to volatile cues of adults, although it responded weakly to chemical trails left on the substrate and to contact chemicals of eggs (Fig. 4). This inconsistency between laboratory and field data may be explained, for example, by a poor adaptation of this parasitoid to M. histrionica, by problems with the parasitoid's taxonomic identity (Gordh \& Beardsley, 1999), or by differences in the behaviour and/or physiology of different parasitoid and/or host strains.

Additionally, the external cues on the eggs of $N$. viridula, E. ventrale and $M$. histrionica appear similar to the parasitoid, as shown by its similar responses during encounter, examination, staying, grooming and probing. On the other hand, discrimination among the different pentatomid eggs depends on internal chemical and/or physical cues, as suggested by higher probing duration and oviposition (acceptance) frequency associated with $N$. viridula eggs.

The new association T. basalis - G. semipunctatum was obtained in the laboratory, as the parasitoid responded to $G$. semipunctatum eggs even more strongly than those of the coevolved host $N$. viridula (Fig. 4), confirming that $G$. semipunctatum is a suitable factitious host for mass rearing of T. basalis (Kartavtsev et al., 1975). Evidence for this comes from the frequency of encounters and examination of $G$. semipunctatum eggs being lower than that of other bug eggs, whereas the examination duration is significantly higher, suggesting that $T$. basalis responds to G. semipunctatum on first encounter, elicited by the chemical compounds present on the eggs. The reason for such a strong response may be the concentration and/or composition of the chemicals involved, which needs to be investigated. This interpretation is strengthened by the fact that both probing and oviposition frequencies for $G$. semipunctatum eggs were higher than for eggs of the natural host $N$. viridula. However, this new association is only partial, because $T$. basalis did not respond to volatiles and responded only weakly to chemical trails of $G$. semipunctatum compared to those of $N$. viridula (Fig. 4). Therefore, on the basis of our data, the probability of this parasitoid adapting to this host in the field appears low. This is confirmed by the fact that, although G. semipunctatum is attacked by other Trissolcus species, T. basalis has never been collected from this species in the field (pers. observ.), nor are there any records of it parasitizing this species in the literature (N.F. Johnson \& L. Musetti, Hymenoptera On-Line Database, http://iris.biosci.ohiostate. edu/hymenoptera/; Salerno, 2000). 
Our explanation of the mechanisms that determine such host-parasitoid associations, however, needs further study of the possible effects of host-induced plant volatiles (Hilker et al., 2002; Colazza et al., 2004a, b; Van Zandt \& Agrawal, 2004), possible behavioural differences among parasitoid strains (Colazza \& Rosi, 2001; Borges et al., 2003), semiochemical differences (geographical dialects) among potential hosts (Aldrich, 1995) and chemical analysis of the compounds involved.

In a recent paper (Conti et al., 2004) it was suggested that host semiochemicals are important in determining host specificity in two species of Trissolcus. Using a different system, Meiners et al. (2000) indicated that specialist parasitoids use cues that are specific to a given system whereas generalists use more general cues. Finally, Del Campo et al. (2003) showed that the feeding specialisation observed in a third model system (plants pea aphid) is determined by behavioural recognition of host specific chemicals, rather than the avoidance of deterrents and/or plant toxins. These results, together with those presented here, support the potential of such an approach for improving our understanding of the mechanisms of host specificity in parasitoids, which may also be applied to the study of predators and herbivores.

ACKNOWLEDGEMENTS. We are grateful to F. De Santis for help in collecting data and to L. Bartoli, D. Fortini, D. Marchionni and A. Luchetti for insect rearing. This work was financially supported by MiPAF "Progetto speciale. Risorse genetiche di organismi utili per il miglioramento di organismi di interesse agrario e per un'agricoltura sostenibile".

\section{REFERENCES}

AldRICH J.R. 1995: Chemical communication in the true bugs and parasitoid exploitation. In Cardè R.T. \& Bell W.J. (eds): Chemical Ecology of Insects 2. Chapman \& Hall, New York, pp. 318-363.

Bin F., Vinson S.B., Strand M.R., Colazza S. \& Jones W.A. 1993: Source of an egg kairomone for Trissolcus basalis, a parasitoid of Nezara viridula. Physiol. Entomol. 18: 7-15.

Borges M., Colazza S., Ramirez-Lucas P., Chauhan K.R., Moraes M.C.B. \& Aldrich J.R. 2003: Kairomonal effect of walking traces from Euschistus heros (Heteroptera: Pentatomidae) on two strains of Telenomus podisi (Hymenoptera: Scelionidae). Physiol. Entomol. 28: 349-355.

Buschman L.L. \& Whitcomb W.H. 1980: Parasites of Nezara viridula (Hemiptera: Pentatomidae) and other Hemiptera in Florida. Fla Entomol. 63: 154-162.

Colazza S. \& Rosi M.C. 2001: Differences in the searching behaviour of two strains of the egg parasitoid Telenomus busseolae (Hymenoptera: Scelionidae). Eur. J. Entomol. 98: $47-52$.

Colazza S., Rosi M.C., Sebastiani P. \& Ursini M. 1996: Host acceptance behavior in the egg parasitoid Trissolcus basalis (Hymenoptera: Scelionidae). Acta Oecol. 17: 109-125.

Colazza S., Salerno G. \& Wajnberg E. 1999: Volatile and contact chemicals released by Nezara viridula (Heteroptera: Pentatomidae) have a kairomonal effect on the egg parasitoid Trissolcus basalis (Hymenoptera: Scelionidae). Biol. Control 16: $310-317$.

Colazza S., Fucarino A., Peri E., Salerno G., Conti E. \& Bin F. 2004a: Insect oviposition induces volatile emission in her- baceous plants that attracts egg parasitoids. J. Exp. Biol. 207: $47-53$.

Colazza S., McElfresh J.S. \& Millar J.G. 2004b: Identification of volatile synomones, induced by Nezara viridula feeding and oviposition on bean spp., that attract the egg parasitoid Trissolcus basalis. J. Chem. Ecol. 30: 945-964.

Conti E., Salerno G., Bin F., Williams H.J. \& Vinson S.B. 2003: Chemical cues from Murgantia histrionica eliciting host location and recognition in the egg parasitoid Trissolcus brochymenae. J. Chem. Ecol. 29: 115-130.

Conti E., Salerno G., Bin F. \& Vinson S.B. 2004: The role of host semiochemicals in parasitoid specificity: a case study with Trissolcus brochymenae and Trissolcus simoni on pentatomid bugs. Biol. Control 29: 435-444.

Del Campo M.L., Via S. \& Caillaud M.C. 2003: Recognition of host-specific chemical stimulants in two sympatric host races of the pea aphid Acyrthosiphon pisum. Ecol. Entomol. 28: 405-412.

FAO 1995: Code of Conduct for the Import and Release of Exotic Biological Control Agents. International Standards for Phytosanitary Measures (ISPM). Publication No. 3, FAO, Rome.

Gordh G. \& Beardsley J.W. 1999: Taxonomy and biological control. In Bellows T.S. \& Fisher T.W. (eds): Handbook of Biological Control. Academic Press, San Diego, pp. 45-55.

Hågvar E.B. 1991: Ecological problems in the establishment of introduced predators and parasites for biological control. Acta Entomol. Bohemoslov. 88: 1-11.

Hilker M. \& Meiners T. 2002: Induction of plant responses to oviposition and feeding by herbivorous arthropods: a comparison. Entomol. Exp. Appl. 104: 181-192.

Hilker M., Rohfritsch O. \& Meiners T. 2002: The plant's response towards insect egg deposition. In Hilker M. \& Meiners T. (eds): Chemoecology of Insect Eggs and Egg Deposition. Blackwell, Berlin, pp. 205-233.

Kartavtsev N.I., Voronin K.E., Sumarakov A.F., Dzyuba Z.A. \& Pukinskaya G.A. 1975: Investigations over many years on the seasonal colonisation of telenomines in the control of the noxious pentatomid in the Krasnodar region. Zash. Rast. 44: 83-90 [in Russian, English abstr.].

JoNES W.A. 1988: World review of the parasitoids of the Southern Green Stink Bug, Nezara viridula (L.) (Heteroptera: Pentatomidae). Ann. Entomol. Soc. Am. 81: 262-273.

Jones W.A., Shepard B.M. \& Sullivan M.J. 1996: Incidence of parasitism of pentatomid (Heteroptera) pests of soybean in South Carolina with a review of studies in other states. J. Agr. Entomol. 13: 243-263.

LEgNER E.F. \& Bellows T.S. 1999: Exploration for natural enemies. In Bellows T.S. \& Fisher T.W. (eds): Handbook of Biological Control. Academic Press, San Diego, pp. 87-101.

LENTEREN J.C. VAN 1997: Benefits and risks of introducing exotic macro-biological control agents into Europe. OEPP/EPPO Bull. 27: 15-27.

Lenteren J.C. van, Babendreier D., Bigler F., Burgio G., HoKKANEN H.M.T., Kuske S., Loomans A.J.M., MenzlerHokkanen I., Rijn P.C.J. van, Thomas M.B., Tommasini M.G. \& ZENG Q.Q. 2003: Environmental risk assessment of exotic natural enemies used in inundative biological control. Biocontrol 48: 3-38.

Meiners T., Westerhaus C. \& Hilker M. 2000: Specificity of chemical cues used by a specialist egg parasitoid during host location. Entomol. Exp. Appl. 95: 151-159.

Nechols J.R., Kauffman W.C. \& Schaefer P.W. 1992: Significance of host specificity in classical biological control. In: Kauffman W.C. \& Nechols J.E. (eds): Selection Criteria and Ecological Consequences of Importing Natural Enemies. Pro- 
ceedings Thomas Say Publications in Entomology, Lanham, pp. 41-52.

Noldus Information Technology 1997: The Observer, System for Collection and Analysis of Observational Data. Reference manual. Version 4.0 for Windows. Noldus Information Technology.

NordLund D.A. 1994: Habitat location by Trichogramma. In Wajnberg E. \& Hassan S.A. (eds): Biological Control with Egg Parasitoids. CABI International, Wallingford, pp. $155-164$

Powell W. 1999: Parasitoid hosts. In Hardie J. \& Minks A.K. (eds): Pheromones of Non Lepidopteran Insects Associated with Agricultural Plants. CABI International, Wallingford, pp. 405-427.

SAFAVI M. 1968: Étude biologique et écologique des hymenoptères parasites des oeufs des punaises des céréales. Entomophaga 13: 381-495.

SAlerno G. 2000: Evaluation of Host Specificity in Pentatomid Parasitoids through their Response to the Host Unit. Ph.D. Thesis, University of Perugia.

Schмidt J.M. 1994: Habitat recognition and acceptance by Trichogramma. In Wajnberg E. \& Hassan S.A. (eds): Biological Control with Egg Parasitoids. CABI International, Wallingford, pp. 165-200.

Siegel S. \& Castellan N.J. JR. 1988: Nonparametric Statistics for Behavioral Sciences. McGraw-Hill, New York, 399 pp.
Statsoft Italia 1997: Statistica per Windows. User's Manual. Statsoft Italia S.r.l.

SteidLe J.L.S. \& Loon J.J.A. van 2002: Chemoecology of parasitoid and predator oviposition behaviour. In Hilker M. \& Meiners T. (eds): Chemoecology of Insect Eggs and Egg Deposition. Blackwell, Berlin, pp. 291-317.

SteidLe J.L.S. \& Loon J.J.A. van 2003: Dietary specialization and infochemical use in carnivorous arthropods: testing a concept. Entomol. Exp. Appl. 108: 133-148.

TodD J.W. 1989: Ecology and behavior of Nezara viridula. Annu. Rev. Entomol. 34: 273-292.

Vet L.E.M. \& Dicke M. 1992: Ecology of infochemical use by natural enemies in a tritrophic context. Annu. Rev. Entomol. 37: 141-172.

VINSON S.B. 1998: The general host selection behavior of parasitoid Hymenoptera and a comparison of initial strategies utilized by larvaphagous and oophagous species. Biol. Control 11: 79-96.

Zandt P.A. van \& Agrawal A.A. 2004: Specificity of induced plant responses to specialist herbivores of the common milkweed Asclepias syriaca. Oikos 104: 401-409.

ZAR J.H. 1999: Biostatistical Analysis. Prentice Hall, Upper Saddle River, NJ, 663 pp.

Received May 24, 2005; revised and accepted November 18, 2005 